\title{
XVIII. An apparatus illustrating crystal forms
}

\section{R. J. Anderson M.A. M.D.}

To cite this article: R. J. Anderson M.A. M.D. (1889) XVIII. An apparatus illustrating crystal forms, Philosophical Magazine Series 5, 28:171, 127-132, DOI: $10.1080 / 14786448908619841$

To link to this article: http://dx.doi.org/10.1080/14786448908619841

$$
\text { 曲 Published online: } 08 \text { May } 2009 .
$$

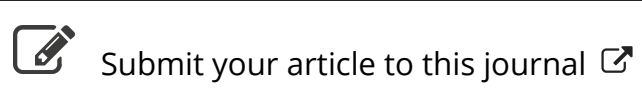

Џll Article views: 2

Q View related articles $\sqsubset$ 
and non-conductors should, on this theory, have their names interchanged. However, such a paradox only arises because one does not specify the kind of conduction or non-conduction considered. Undoubtedly metals are non-conductors of electric force, and just for this reason they compel it under certain circumstances to remain concentrated instead of becoming dissipated, and thus they become conductors of the apparent source of these forces, electricity, to which the usual terminology has reference.

Karlsruhe, March 1889.

XVIII. An Apparatus Illustrating Crystal Forms. By R. J. Anderson, M.A., M.D., Professor of Natural History in Queen's College, Galway*.

\section{[Plate II.]}

THE apparatus by which $I$ propose to illustrate crystal 1 forms consist of frameworks and cords and weights.

The first piece of apparatus is figured in Plate II. fig. 1, and consists of a frame made of wood. This is divided into two compartments. One of these has, above, a slit half an inch wide that runs from end to end ; in this slit a slide moves to and fro, and can be fixed by means of a binding-screw at any place.

A slide of a similar kind moves in a slit in the lower part of the framework; this can be fixed by binding-screws in any position. Pulleys are fixed at the ends of this compartment.

Slips of wood run from end to end at the sides and carry riding-slides. These slides have binding-screws and pulleys whose sheaves revolve on a vertical axis fitted to them.

A figure is easily construeted by carrying cords over the pulleys. Single cords only are shown in the figure. This is for the sake of distinctness.

Starting from $\alpha^{\prime}$, which marks a ring connected with the weight $p$, a single cord runs through $\beta^{\prime}$ (ring), $\eta^{\prime}$ (ring), $\delta^{\prime}$, through ring $\alpha^{\prime}$, through $\eta^{\prime}$, to be fixed to a weight.

A second cord starting from $\gamma^{\prime}$ runs through $\alpha^{\prime}, \epsilon^{\prime}, \eta^{\prime}$, through ring $\gamma^{\prime}$, and then across to $\epsilon^{\prime}$ through this ring to hook up a weight $p^{\prime}$. A third cord is fixed to $\delta^{\prime}$ and runs through $\epsilon^{\prime}, \beta^{\prime}, \gamma^{\prime}$, through $\delta^{\prime}$ and $\beta^{\prime}$ to loop up another weight. The actual tension-weights are fixed to the small rings, which act as pulleys.

Each rhombus has in this way a cord to itself, and the size of the rhombus depends on the weights attached. The smaller the rhombus the more cord is to spare.

The figure shown is the regular octahedron if the axes be

* Communicated by the Physical Society : read A pril 13, 1889. 

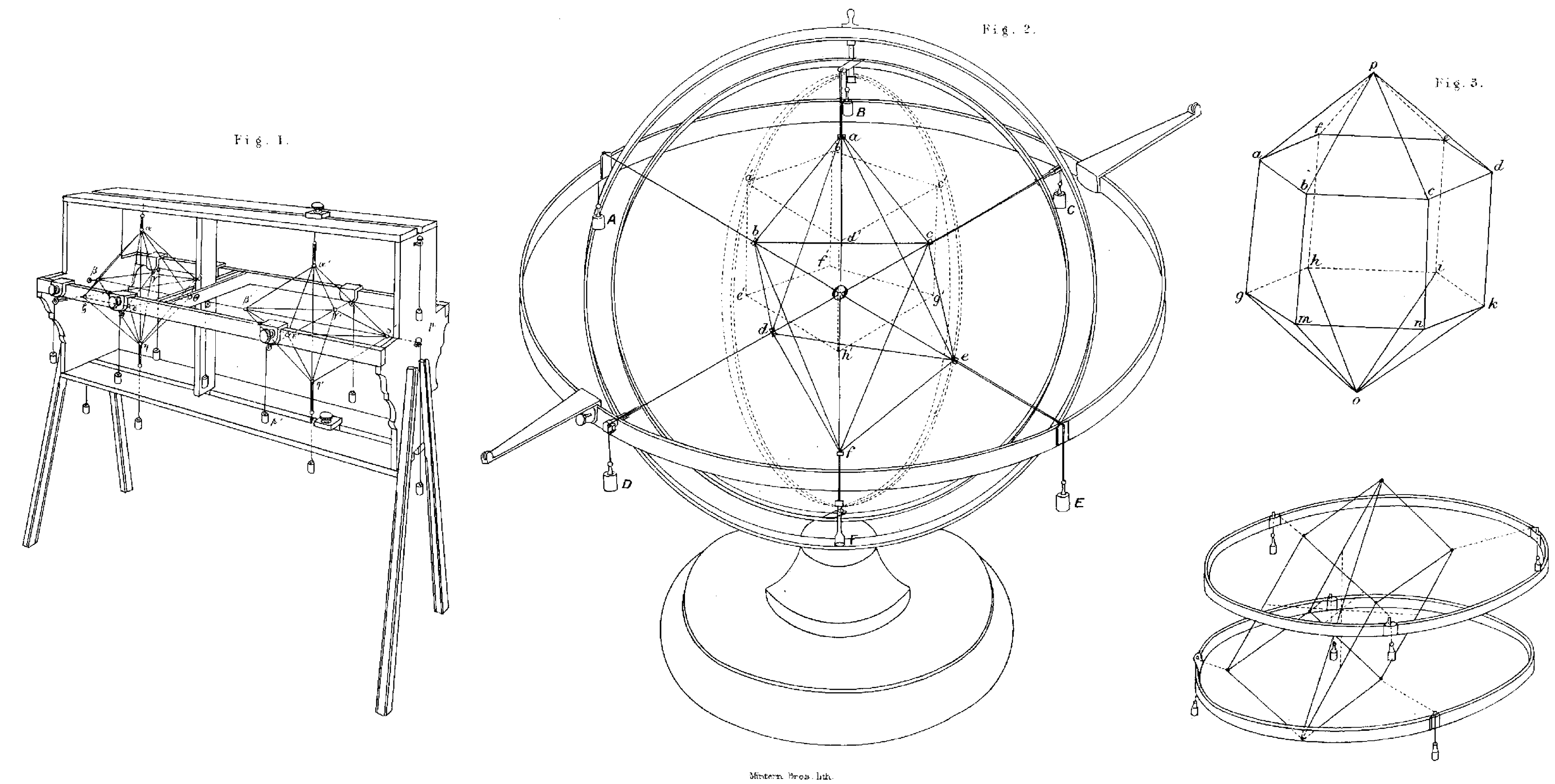
equal. This condition is easily produced by adjusting the weights.

The octahedron of the second dimetric system, or pyramidal system, is produced by increasing the weights above and below.

The octahedron of the third system may be easily formed by increasing a pair of the horizontal weights.

The octahedral figures may be easily formed by leaving out the diagonals and running the cords from the rings at one extremity of the rhombuses through two rings, and then throngh the opposite ring, to be there fixed to a weight. The tension-weights, as shown in the figure, will then correspond to the apices of the rhombuses.

For the oblique systems further changes are necessary. The upper slide is moved to the right and the lower to the left, or vice versê. This is attended with elongation of the vertical axes, and the cords passing through the pulleys above and below and at the ends are increased, and the slack below is pulled in to a less extent. The other sides of the octahedron are less affected.

In the first place, the lateral rider-slides are allowed to remain in a position such that the line joining them is perpendicular to the central vertical longitudinal plane. This gives the Monoclinic System.

Secondly, the rider-slides are moved one to the right, the other to the left, and in this way the Anorthic or Triclinic System is produced.

In each case it is desirable to have the slack for each rhombus at different angles of the octahedron.

All the possible varieties of the fifth system cannot be produced in this way. So it is necessary to arrange for the elevation and depression to the rider-slides in extreme cases. This is accomplished by means of a large ring which carries a pulley.

I have chosen the octahedron as the simplest figure.

The cube is formed by the introduction of two horizontal hoops, one above and one below the level of the horizontal bars. These by a simple mechanism are made movable; and if eight pulleys be fixed opposite the eight edges of the octahedron, and the edges of the octahedron be drawn out by rings running on these cords, it will be necessary, then, only to run cords through rings above and below, and to relax the horizontal and apical weights in order to produce the cube.

The modifications caused by truncating or bevelling the edges or faces can be produced by increasing the number of the hoops or rings. For the simplest figures, however, vertical hoops answer best. The sliding-rings that are carried by the 
cords may with their transverse cords be lowered to the level of the bar again, and the octahedron again produced.

The cube and the corresponding forms of the pyramidal and prisıatic systems may be easily constructed by 1 unning the cords as follows :-

Take the cube as

$$
\begin{array}{llll}
a^{\prime} & b^{\prime} & c^{\prime} & d^{\prime} \\
e^{\prime} & f^{\prime} & g^{\prime} & h^{\prime}
\end{array}
$$

where the first row represents the upper face, and the second the lower, as in fig. 2.

The cords will have the following course :-

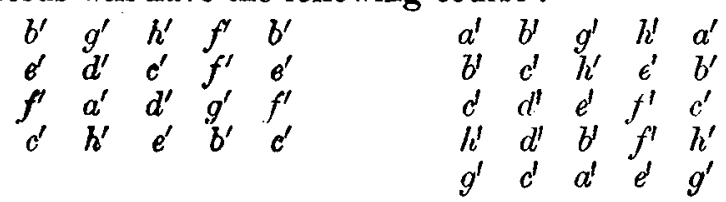

The faces of the cube corresponding to the angle of the octahedron.

Now by drawing out the cords opposite the middle of the faces (that is, the diagonals of the faces) a 24 -sided figure is produced which can be reduced in the limit to a 12 -sided figure, namely the rhombic dodecahedron.

If the sides be connected by cords with pulleys and drawn out, and at the same time cords connecting the centres of the sides with the centres of the faces be drawn out, then the trapezohedron is produced.

Cause the two lateral pulleys of the cube to approach above and the longitudinal pair to approach below, and the tetrahedron is produced. A prism surmounted by pyramids is produced by drawing out the diagonals of the terminal faces; from this the corresponding octahedron may be obtained by a simple method.

The other part of the framework is shown in the drawing (fig. 1, left-hand) as containing the double hexagonal pyramid. Sliding-pulleys, as in the part already described, are fitted above and below. Rider-pulleys are attached to the bars at the sides. Two are shown on each side.

The cords are attached in this way:-A bundle of six are fastened together above to a cord, and drawn by this cord through a ring. The cord passes over two pulleys and reaches a weight outside the framework. The six cords pass through the rings marked $\beta, \gamma, \delta, \zeta, \theta$, and $\epsilon$ in the figure, then down to be attached to a cord below, which goes through a pulleysheaf. The rings marked by the Greek letters are seen attached by cords to weights, through each of these a cord passes. This cord is carried through one of the rings and 
kept there by means of a small weight. The ratio of the vertical to the horizontal axes may be easily altered hy means of the weights. The approximation of the lateral pulley gives rise to the octahedron. The number of the sides may be increased by increasing the cords and pulleys.

In order to show other figures two hoops are fitted to the framework, above and below. The cords of the pyramids are hooked out, and the cords connected with the hooks pass over pulleys and are attached to weights. A cord is made to go through the rings (hook-rings) above and below. By running down the rings and anhooking the weights above and below, the hexagonal prism is produced.

Prisms with more sides can be produced by increasing the number of the cords, which correspond to the edges. The pyramids surmounting the prism are produced by drawing out the cords at the extremities of the prism. Figures with fewer sides are produced by causing the pulleys to approach. Forms the result of bevelling and truncation are produced by pulling out the cords of the terminal pyramids and running other cords through the rings. The original double six-sided prisms are produced by causing the hoops to approach one another.

The ikosahedron is produced by forming the five-faced equilateral pyramid above and below, and approaching the hoops towards one another, so that the distance between the hoops is equal to the perpendicular of one of the triangles. Then it is only necessary to rotate the lower hoop though $36^{\circ}$, and to connect the obtuse angles of the rhombus. In this way the figure can be produced.

The relations of the hexagonal to the rhombohedral division of the sixth system may be shown in this way. Take the double pyramid, hook up each alternate horizontal angle, and hook down the others. Adjust suitably the superior and inferior angles, and the rhombohedron is produced. The cords in reality follow the course of the lines in the glass models.

This method is very interesting in this way, that by a little dodging the rhombohedron can be converted into the cube, so that the relations between the sixth and other systems are rendered more distinctly apparent.

The rhombohedron may be easily changed into the hexahedron by unhooking the weights and puliing in the cord. The hoops are shown in the lower part of fig. 3, Pl. II., with the rhombohedron attached. The hexagonal prism is figured separately for the sake of distinctness.

The ikosahedron may be produced by hooking up and down the horizontal cords of the decahedral pyramids. If 
we begin with the double octahedral pyramids, the rhombio dodecahedron can be easily produced by hooking ap the corresponding alternate edges above and below, and running cords through the hooks looped up and those rings still stationary.

In order to show the effects of uniting and separating forces the form shown at fig. 2 is useful. The instrument consists of a frame in which hoops revolve, some on vertical and others on horizontal axes. The hoops carry sliding-pulleys as shown on the plate. The cube is easily constructed by running cords over eight pulleys fixed on two rings revolving on a vertical axis. Cords are carried through small rings above and below (fig. 2, $a^{\prime} b^{\prime} e^{\prime} d^{\prime} e^{\prime} f^{\prime} g^{\prime} h^{\prime}$ ).

Withont going into details, it will be easily seen that one orthogonal hexahedron can be easily changed into another, and into the corresponding octahedron. The octahedron of the first system, $a b c d e f$, if constructed by running cord over the pulley $\mathrm{B}$, and the pulley attached to the same ring below, may be changed into the octahedron of the dimetric or trimetric system, or of either of the oblique. The latter is accomplished by causing the hoop to revolve, and for the triclinic the vertical hoops come into action. Adjustment of the weights leads to an alteration in the axes, and the relations of the weights for a special form may be studied.

It is evident that the dodecahedron and trapezohedron may be produced in this instrument as in the first, and that the forms due to truncating or bevelling of the sides are obtained very readily.

The following are the advantages of the apparatus:-First, it shows clearly the effect of changes of force in producing changes of form. The weights can be approximated or separated, and thus the relations of allied forms may be studied. The number of weights may be increased, and the change of form by grouping may in this way be well shown.

If we take an india-rubber tissue ball inflated with air as an example of an infinite number of forces acting from a centre, and a piece of stretched cord with a weight attached as the other extreme limit, many of the intermediate conditions where strings are made to form the edges of figures may be easily understood from the arrangements I have described.

It is true that such methods as are here suggested are open to the objection that mathematical principles of a very important kind are involved. I think the same objection may bo made to any mechanical contrivance; but so far from getting rid of a difficulty without explaining it, I bold that the apparatus, whilst it will produce a better conception of crystal forms, and the actual work in the crystals themselves, 
in the minds of those students who know very little about mathematics, viz., almost all students of chemistry and mineralogy, and a still larger number of geological students, the apparatus will prove useful to mathematical students inasmuch as the arithmetical processes are tedious and complex for even those forms in which the mathematical relations are comparatively simple. For the forms with oblique axes the advantages of a simple method of noting the weights necessary to maintain equilibrium far outweigh the disadvantages.

Note.-Professor Wiltshire informs me that many years ago, Mr. Mitchell, at the Royal Institution, showed a model by which the derivation of the crystalline systems from the octahedron was explained.

\section{Explanation of Plate $\Pi$.}

Fig. 1.- $\alpha^{\prime}, \beta^{\prime}, \gamma^{\prime}, \delta^{\prime}, \epsilon^{\prime}, \eta^{\prime}$, octahedron.

$$
p, p^{\prime} \text {, weights. }
$$

$\alpha, \beta, \gamma, \delta, \epsilon, \zeta, \eta, \theta$, double hexagonal pyramid.

Fig. 2,- $-a, b, c, d, e, f$, octahedron. $a^{\prime}, b^{\prime}, c^{\prime}, d^{\prime}, e^{\prime}, f^{\prime}, g^{\prime}, h^{\prime}$, cube.

$A, B, C, D, E, F$, weights.

Fig. 3.-The upper figure shows the hexagonal prism surmounted by hexagonal pyramids. The lower shows the rings with the rhombohedron formed.

XIX. A Shunt-Transformer. By Mr. E. W. Smiti*".

A LTHOUGH this experiment has already been described A by Professors Ayrton and Perry in a paper at the Institution of Electrical Engineers, it was thought to be worth while occupying this Society's time in showing it here, as it forms a good lecture-experiment, if nothing more, to illustrate acceleration and lag of alternating currents.

The experiment consists as follows :-Between two leads a

Fig. 1.

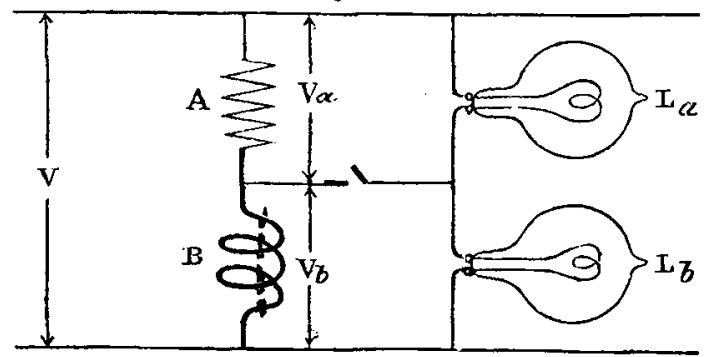

certain alternating potential difference, $\mathrm{V}$, is maintained. We have two resistances, $A$ and $B$ (fig. 1), in series, through

* Cnmmunicated by the Physical Society: read June 8, 1889. 